\title{
Hydrodynamic instabilities as a source of variability in AGN jets
}

\author{
I.P. van der Westhuizen ${ }^{* a}{ }^{a}$ B. van Soelen, ${ }^{a}$ and P.J. Meintjes ${ }^{a}$ \\ ${ }^{a}$ University of the Free State \\ Bloemfontein, South Africa \\ E-mail: vanderwesthuizenip@ufs.ac.za, vansoelenb@ufs.ac.za, \\ meintjpjeufs.ac.za
}

\begin{abstract}
Most radio-loud AGN are associated with jet-like structures that can extend over hundreds of kiloparsecs. These jets are a source of variable emission that covers most of the electromagnetic spectrum. The dominant component of the emission is produced through non-thermal processes like synchrotron radiation. In this study we investigate the contribution of hydrodynamic instabilities to the long term variability observed within these sources. This is done by undertaking 3D hydrodynamic simulations of a relativistic jet that is evolved with time. The simulation is constructed with the hydrodynamic code PLUTO and consists of a rectangular grid, spanning $256 \times 256 \times 512$ cells. The environment contains a uniform background medium into which less dense jet material is injected, at a Lorentz factor of 10 . We have developed a post-processing code in order to determine the synchrotron emission that will be produced by this environment and calculate intensity maps at arbitrary viewing angles with respect to the hydrodynamic environment. In this code we assume that the emission is produced by non-thermal electrons in a power-law distribution and take into account geometric and relativistic effects. The resulting intensity maps show a similar large scale morphology to that of FR II type AGN, containing a central relativistic beam surrounded by lobe structures. The results also show the formation of time dependent structures, such as knots and blobs, due to hydrodynamic instabilities. It was found that these structures may cause a variation of up to $10 \%$ in the total intensity.
\end{abstract}

5th Annual Conference on High Energy Astrophysics in Southern Africa

4-6 October, 2017

University of the Witwatersrand (Wits), South Africa

${ }^{*}$ Speaker. 


\section{Introduction}

The jets associated with radio-loud active galactic nuclei (AGN) have complex morphologies that show time dependent emission components. These jets are a result of relativistic plasma ejected from the central engine of the AGN and can stretch up to hundereds of kiloparsecs (see e.g. [1]). Several long term monitoring programs have been undertaken to study the kinematics of these jets focusing on the proper motions of emission regions (see e.g. [2] and [3]). These studies have shown a wide range of apparent motions for emission regions, ranging from stationary up to superluminal speeds of $\sim 70 c$. The formation and evolution of different emission components within these jets are not fully understood but it is likely a combination of several factors such as the properties of the ejected plasma as well as the surrounding medium in which the jet is formed.

In combination with observational studies, hydrodynamic simulations can be a powerful tool to investigate the kinematics of AGN. Many studies have investigated the formation and evolution of relativistic jets with numerical simulations. One of the first in-depth parameter studies to investigate the effects of different parameters on the morphology of relativistic jets was showed by Martí et al. [4]. This study investigated the effects of different density ratios between the jet and ambient medium and Lorentz factors. In particular it was shown that jets with larger Lorentz factors have a greater propagation efficiency and less turbulent instabilities.

Subsequent studies have been done to investigate the formation and evolution of time dependent components within jets. Agudo et al. [5] showed that a series of emission components can be generated by a single perturbation in the flow of the beam. The perturbation in the beam forms re-collimation shocks which are observed as trailing components varying in brightness as well as apparent velocity. Aloy et al. [6] showed that superluminal components may also be produced by the precessing of the jet.

In this study we will investigate the effects of fluid dynamic instabilities, such as KelvinHelmholtz and Rayleigh-Taylor instabilities, on the emission of relativistic jets and whether these types of instabilities could produce individual emission components without a perturbation to the injection of the jet fluid. This is done by first constructing a 3D simulation of a relativistic jet (section 2) and then modelling the synchrotron emission that would be produced in the simulated environment (section 3).

\section{Hydrodynamic model}

A relativistic hydrodynamic simulation of an AGN jet was created and evolved with time using the PLUTO (v4.2.) code [7]. The code uses high resolution shock capturing (HRSC)-Godunov schemes to evolve the fluid dynamic conservation equations, given by

$$
\frac{\partial \vec{U}}{\partial t}=-\nabla \cdot \overrightarrow{\vec{T}}(\vec{U})+\overrightarrow{\vec{S}}(\vec{U})
$$

with time. Here $\vec{U}$ is a vector that contains the properties that are conserved, $\vec{T}(\vec{U})$ is a tensor containing the flux vectors as a function of $\vec{U}$, and $\overrightarrow{\vec{S}}(\vec{U})$ is a tensor containing source terms that can be used to introduce effects such as viscosity and gravitational forces [7]. 


\begin{tabular}{ll}
\hline Parameter & Value \\
\hline Lorentz factor $(\Gamma)$ & 10 \\
Jet density $\left(\rho_{j}\right)$ & $10^{-1}$ \\
Ambient density $\left(\rho_{a}\right)$ & $10^{4}$ \\
Pressure $(P)$ & $10^{-3}$ \\
Mach number & 7.8 \\
Adiabatic index $\left(\gamma_{b}\right)$ & $5 / 3$ \\
\hline
\end{tabular}

Table 1: Parameters assigned to simulation, density and pressure are given in arbitrary units.

In this study we assume that the distance between the simulated jet and the central engine of the AGN is large enough such that the jet would be kinetically dominated and that the magnetic field would not have a dynamic effect on the large scale structure. Under this assumption the relativistic hydrodynamic (RHD) module in PLUTO was used to evolve the environments [7]. No source terms were included in this simulation (i.e. $\overrightarrow{\vec{S}}(\vec{U})=0$ ).

The computational domain of the simulation consisted of a three dimensional Cartesian grid spanning $256 \times 256 \times 512$ cells. A fluid at rest with uniform density and pressure was assigned to the grid for the background medium. A circular nozzle with a radius of 8 cells was constructed on the lower $z$-boundary and used to inject material into the domain. The injected material consisted of a less dense medium with an initial Lorentz factor of 10. A profile was used for the nozzle to ensure a smooth transition between the jet and ambient medium. The parameters that were used for the simulation are listed in table 1. Arbitrary units were used for the density and pressure to avoid unnecessary truncation errors. The small density ratio $\left(\eta=10^{-5}\right)$ between the ambient and jet medium was chosen to promote the formation of instabilities in the flow. The injected material had a supersonic bulk flow of Mach 7.8. The parameters were chosen to produce a pressure equilibrium between the jet and the ambient medium.

In order to evolve the environment with time the Piecewise parabolic method was used in conjunction with the HLLC Riemann solver [8]. The boundary conditions of the simulation were set to outflow for all boundaries except the lower $z$ boundary, which was set-up to be reflective. The simulation was evolved until the shock between the ambient and jet medium reached the edge of the computational domain.

\section{Synchrotron emission}

To investigate the time dependent emission that forms in the simulation due to fluid dynamic instabilities, a post-processing code was designed to estimate the synchrotron emission. Synchrotron emission is the dominant radiative process at radio frequencies at which most of the kinematic studies have been done. In the model we assumed that the dominant radiating particles would be relativistic non-thermal electrons in a single power-law distribution given by:

$$
n_{\mathrm{e}}(\gamma)=n_{0} \gamma^{-p},
$$

where $\gamma$ is the Lorentz factor, $n_{\mathrm{e}}(\gamma)$ is the number density of electrons as a function of Lorentz factor, $p=1.8$ is the particle index and $n_{0}$ is a normalization factor. It was assumed that the non- 
thermal electrons were in equilibrium with the energy density of the fluid and the normalization parameter is calculated as [9],

$$
n_{0}=\left[\frac{\mathscr{U}(p-2)}{1-C_{E}^{2-p}}\right]^{p-1}\left[\frac{1-C_{E}^{1-p}}{\mathscr{N}(p-1)}\right]^{p-2},
$$

where $\mathscr{U}$ is the electron energy density, $\mathscr{N}$ is the electron number density (it was assumed that the fluid consisted purely of protons and electrons) and $C_{E}$ is the ratio of the maximum and minimum electron energy.

The synchrotron emission and absorption coefficients were calculated for each cell in the simulation using a delta approximation, which assumes that each electron in the distribution radiates at a single frequency. The coefficients were calculated according to the following equations [10],

$$
j_{v}^{\mathrm{em}}=\frac{4}{9}\left(\frac{e^{2}}{m_{\mathrm{e}} c^{2}}\right)^{2} u_{B} c v^{1 / 2} v_{0}^{-3 / 2} n_{\mathrm{e}}\left(\sqrt{\frac{v}{v_{0}}}\right),
$$

and

$$
\alpha_{v}^{\mathrm{em}}=\frac{2}{9} \frac{p+2}{m_{\mathrm{e}}}\left(\frac{e^{2}}{m_{\mathrm{e}} c^{2}}\right)^{2} u_{B} c v^{-2} v_{0}^{-1} n_{\mathrm{e}}\left(\sqrt{\frac{v}{v_{0}}}\right) .
$$

Here $j_{v}^{\mathrm{em}}$ and $\alpha_{v}^{\mathrm{em}}$ are the emission and absorption coefficients in the co-moving frame of the fluid, $e$ is the charge of an electron, $m_{\mathrm{e}}$ is the mass of an electron, $c$ is the speed of light, $u_{B}$ is the magnetic energy density, $v$ is the frequency in the co-moving frame and $v_{0}$ is given by,

$$
v_{0}=\frac{3 e B}{4 \pi m_{\mathrm{e}} c} .
$$

The magnetic energy density was calculated assuming equipartition with the internal energy of the fluid.

In order to calculate $2 \mathrm{D}$ intensity maps from the $3 \mathrm{D}$ environment, radiative transfer equations were applied and the emission and absorption coefficients were integrated along a line of sight. The line of sight can be arbitrarily defined and the relevant Lorentz transformations were taken into account during the integration. The radiative transfer equations can be expressed as,

$$
\frac{\mathrm{d} I_{v^{\text {rec }}}^{\mathrm{rec}}}{\mathrm{d} s}=\delta^{2} j_{v}^{\mathrm{em}}-\delta^{-1} \alpha_{v}^{\mathrm{em}} I_{V^{\mathrm{rec}}}^{\mathrm{rec}},
$$

where,

$$
\delta=\frac{1}{\Gamma(1-\beta \mu)},
$$

is the Doppler factor of the bulk flow, with $\mu=\cos (\phi)$ representing the angle between the motion of the bulk flow and the line of sight, $\Gamma$ the Lorentz factor and $\beta=v / c$ the velocity of the bulk flow. The light travel time and cosmological redshift were neglected in the calculations.

By integrating the intensity over the entire map relative light curves can be constructed of the evolution of the system. These light curves show the variation in the total intensity of the system rather than specific emission regions. While the results neglect light travel time and cosmological redshift it provides a first approximation on whether the instabilities that formed in the simulated jet can produce significant variability without the injection of additional perturbations. 


\section{Results}

The physical structure of the simulated jet presented in this paper has been discussed in detail in previous contributions [11]. Here we will show the variation in emission produced by fluid dynamic instabilities. Figure 1 shows intensity maps plotted for a viewing angle perpendicular to the injection direction at several time steps. The dominant emission originates from the cocoon of the jet. The cocoon is a turbulent region containing a mixture of jet and ambient material, which surrounds the relativistic beam. It is formed by the backflow of jet materiel after it has reached the interface between the beam and the surrounding medium. This structure is similar to that of FR II type radio galaxies. The results show the presence of hots spots and filaments, which change significantly over time.

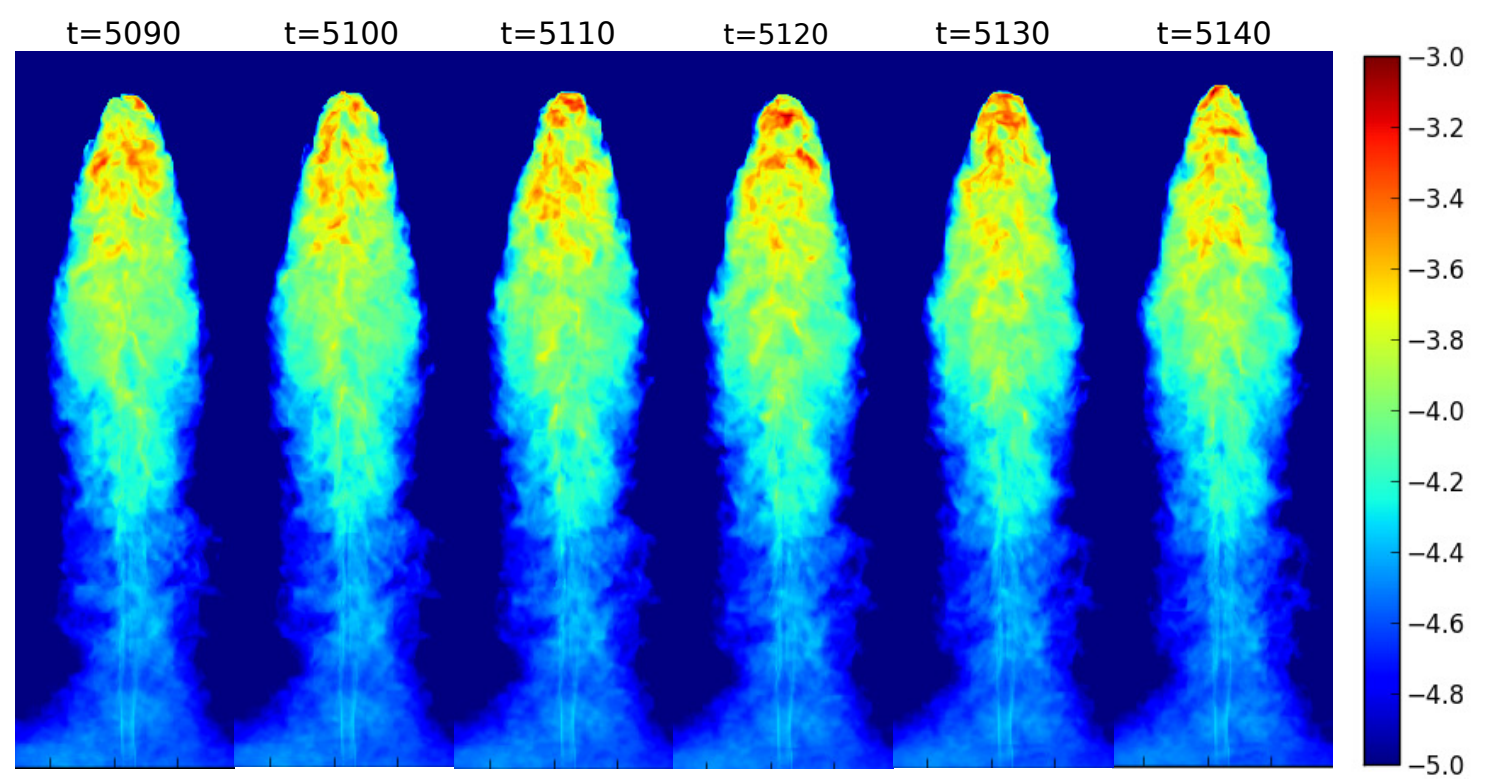

Figure 1: Two dimensional intensity maps calculated for a viewing angle of $90^{\circ}$ with respect to the axis of injection of the jet. The intensity maps are plotted for consecutive time steps from 5090 to 5140 , with equal time intervals $\Delta t=10$, in arbitrary time units.

To investigate the time dependence of emission structures within the jet beam, intensity maps were calculated at time intervals between $t=5090$ and $t=5140$, at an angle of $15^{\circ}$ relative to the axis of injection of the jet (Figure 2). At such a low viewing angle the emission in the jet becomes Doppler boosted and dominates over the cocoon emission, emphasizing the jet structure. The results show the presence of both stationary and time dependent emission regions in the jet. The stationary components are formed in the stable region of the beam by re-collimation shocks, while the time dependent structures form where the beam of the jet becomes unstable and breaks apart. The position of a moving component is marked in Figure 2 versus that of a stationary component close to the injection site.

Due to the large time difference between the write out of data files in the fluid dynamic simulation when compared to the bulk motion of the jet medium, it is not possible to determine whether the change in the top emission region is the result of a single component propagation through the 
jet or multiple emission components. This combined with the neglect of light travel time in the post-processing code makes determining the apparent velocity highly challenging.

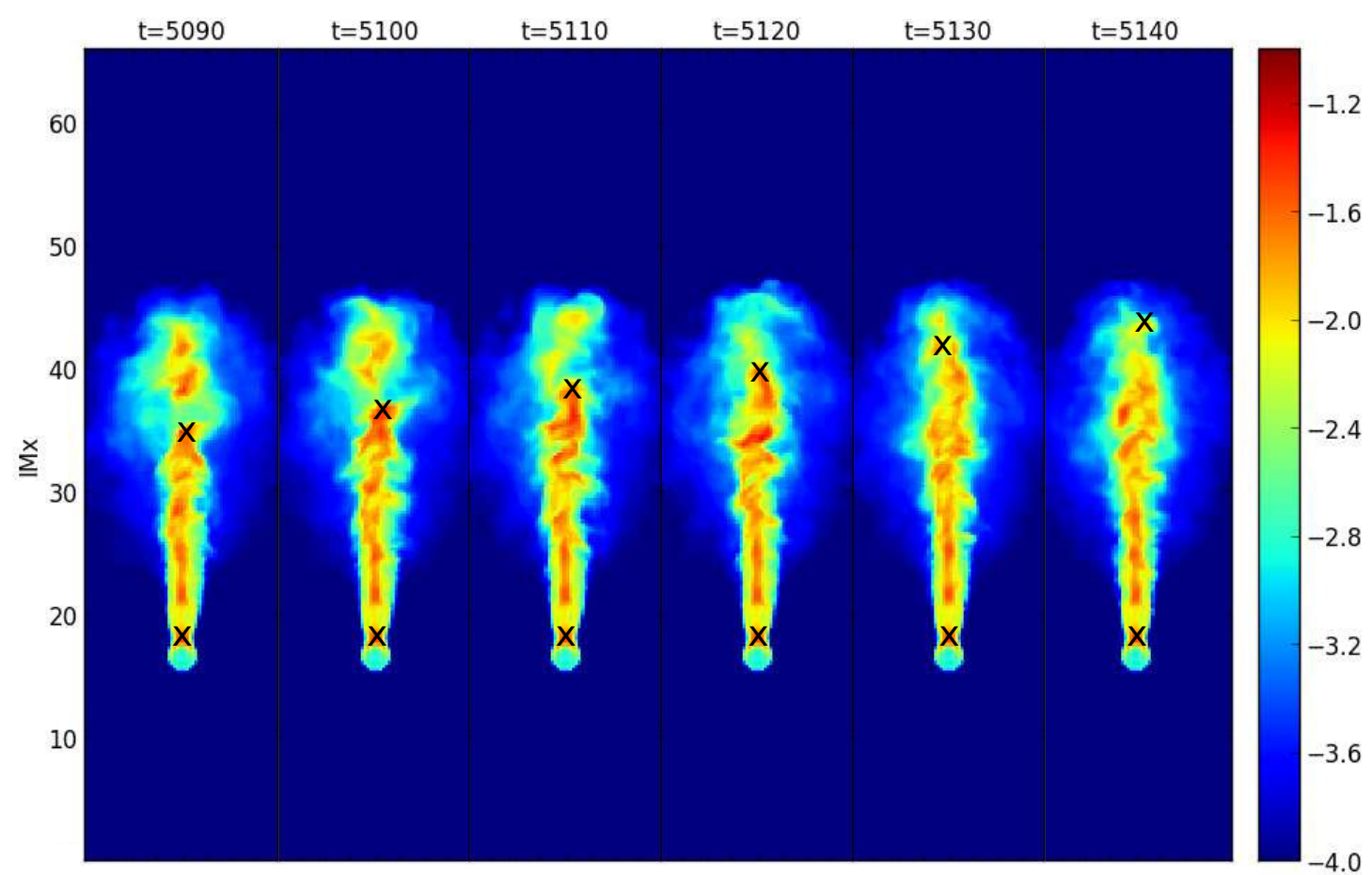

Figure 2: Two dimensional intensity maps calculated for a viewing angle of $15^{\circ}$ relative to the axis of injection of the jet. The intensity maps are plotted for consecutive time steps from 5090 to 5140, with equal time intervals $\Delta t=10$, in arbitrary time units. Crosses mark the positions of two emission components, one stationary and the other propagating along the beam.

The variation in total intensity with time is shown in Figure 3. For a face-on system (viewing angle of $0^{\circ}$ relative to the axis of injection of the jet) the relative flux increases at a high rate initially, but slows down at later time steps with a clear break in the curve. The initial increase in flux is due to an increase in the beam length (which is the dominant emission component in the face-on source) as the jet propagates through the medium. After the beam reaches a certain length $(\approx 80$ units) it becomes unstable and breaks apart. The beam does not increase in length past this point and the rate at which the flux increases slows down. For an edge-on source (viewing angle of $90^{\circ}$ relative to the axis of injection of the jet) the relative flux shows a steady increase. In this case the dominant emission component is the cocoon, which increases at a steady rate as more jet material is injected.

To investigate the scale of short term variability within the light curve a running mean curve was constructed. Each point on the running mean consisted of the mean of the 25 points before and 

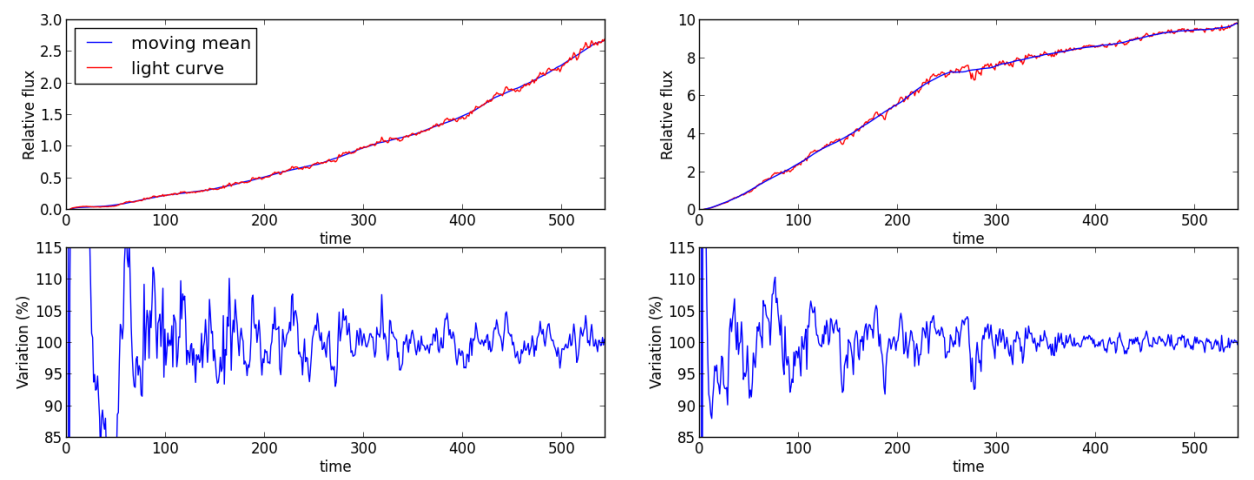

Figure 3: Relative light curves for edge-on (left) and face-on (right) lines-of-sight with respect to the jet propagation, showing the evolution of flux for the simulations. The top plots show the relative flux for each intensity map as well as for a moving mean of 50 frames. The bottom plots contains the percentage variation in flux with respect to the moving mean.

after the current time step,

$$
\bar{y}_{i}=\frac{\sum_{j=i-25}^{25} y_{j}}{50} .
$$

The difference between the relative light curve and the running mean curve was calculated as a percentage value in order to determine the small scale variation in flux. The variation light curves show small variability for both lines-of-sight, with the percentage variability decreasing as the flux increases. The face-on source produces a lower percentage variation than that of the edgeon source, however, the relative flux of this source is much higher and, therefore, the magnitude of these variations are larger. Overall these light curves show that variability can be produced through the fluid dynamic instabilities which are generated within AGN jets without the need for a perturbation in the injection rate. The amplitude of the variations remain similar throughout the simulation, while the overall flux increases, which results in a decline of the percentage variation. No large scale flares were observed in the light curves suggesting that a perturbation in the injection parameters is necessary to produce the flares which have been associated with observed data.

\section{Conclusion}

In this study a 3D relativistic hydrodynamic simulation was created that is consistent with the results shown by previous studies. Synchrotron emission modelling was applied to calculate artificial intensity maps at $15 \mathrm{GHz}$. These maps showed the production of stationary as well as moving emission components at a low inclination angles. This result shows that fluid dynamic instabilities actively contribute to the emission structure observed within AGN jets.

Total intensity light curves were calculated and showed a general increase in brightness as the jet evolved. This was due to the increased size of the jet within the simulation with time. The light curves showed that different inclination angles lead to different features in the evolution of the intensity, which is due to different emission regions that dominate at different viewing angle 
due to Doppler boosting. Small scale variability was shown with respect to the mean intensity. The amplitude of the variation reached on the order of $10 \%$ of the total intensity of the jet, however the percentage decreased with the age of the system. This result shows that fluid dynamic instabilities may contribute to the overall variability of AGN jets but scale of the variations shown in this study is much less than that observed for flares in these sources. This suggests that additional perturbations are required to produce the large amplitude variability which has been observed.

This study provides a good initial investigation, however the accuracy will be increased for future investigations. This will be done by including additional effects such as light travel time and radiative cooling of electrons. This will allow us to decrease the time resolution of the variability as well as measure additional properties such as the velocity of the apparent motion of emission regions.

\section{References}

[1] J. Bagchi, et al., Megaparsec Relativistic Jets Launched from an Accreting Supermassive Black Hole in an Extreme Spiral Galaxy, ApJ 788, pp. 174, (2014)

[2] M.L. Lister, et al., MOJAVE: XIII. Parsec-scale AGN Jet Kinematics Analysis Based on 19 years of VLBA Observations at $15 \mathrm{GHz}, A J$ 125, pp. 12, (2016) [doi: 10.3847/0004-6256/152/1/12]

[3] S. G. Jorstad, et al., Kinematics of Parsec-scale Jets of Gamma-Ray Blazars at $43 \mathrm{GHz}$ within the VLBA-BU-BLAZAR Program, APJ 846, pp. 35, (2017) [doi: 10.3847/1538-4357/aa8407]

[4] J. M. Martí, E. Müller, J. A. Font, J. M. Ibáñez and Marquina Morphology and dynamics of relativistic jets, ApJ 479, pp. 151, (1997) [doi: 10.1086/303842]

[5] I. Agudo, et al., Jet Stability and the Generation of Superluminal and Stationary Components ApJ 549, pp. L183-L186, (2001) [doi: 10.1086/319158]

[6] M. Aloy, et al., Three-dimensional Simulations of Relativistic Precessing Jets Probing the Structure of Superluminal Sources ApJ, 585, pp. L109-L112, (2003) [doi: 10.1086/374543]

[7] A. Mignone et al., PLUTO: a numerical code for computational astrophysics, ApJS 170 (2007) 228 [doi: 10.1086/513316]

[8] A. Mignone and G. Bodo, An HLLC Riemann solver for relativistic flows - I. Hydrodynamics, MNRAS 364 (2005) 126 [doi: 10.1111/j.1365-2966.2005.09546.x]

[9] J.L. Gómez et al., Parsec-Scale Synchrotron Emission from Hydrodynamic Relativistic Jets in Active Galactic Nuclei, ApJ 449 (1995) L19 [doi: 10.1086/309623]

[10] M. Böttcher, D.E. Harris and H. Krawczynski. (Eds.), Relativistic Jets from Active Galactic Nuclei, Wiley-VCH 2012

[11] I.P. van der Westhuizen, B. van Soelen, P.J. Meintjes, J. Beall, Hydrodynamics and Instabilities of Relativistic Astrophysical Jets in AGN Frontier Research in Astrophysics II., PoS(FRAPWS2016)081 